\title{
The Optimization of Solar Drying of Grain by Using a Genetic Algorithm
}

\author{
M. M. RAHMAN ${ }^{1}$, A. G. M. B. MUSTAYEN ${ }^{1}$, SAAD MEKHILEF $^{1}$, and R. SAIDUR ${ }^{2}$ \\ ${ }^{1}$ Department of Electrical Engineering, Power Electronics and Renewable Energy Research Laboratory, University of Malaya, \\ Kuala Lumpur, Malaysia \\ ${ }^{2}$ Department of Mechanical Engineering, University of Malaya, Kuala Lumpur, Malaysia
}

Particularly in the low drying temperature drying is an energy intensive process and requires a suitable optimization technique. This work represents the kinetic simulation and energy efficient optimization technique for drying long grain, i.e. rice. At $30-60^{\circ} \mathrm{C}$ temperature the grains have been dried in a solar powered air dryer. After certain time interval the enzymatic activity and the moisture content have been measured. Genetic Algorithm (GA) has been used for the simulation and the optimization process while the experimental data have been used to fit the thin layer drying model. The results indicate that between 40 and $50^{\circ} \mathrm{C}$ temperature and the $1.3-1.5 \mathrm{~m} / \mathrm{s}$ air flow rate the drying time for the rice is around $120-180 \mathrm{~min}$. The kinetic simulation and the quality analysis confirm that the dried products have a better enzymatic activity which resembles the quality standard and the required energy for the optimized drying process is lower than the others.

Keywords: Drying process, Enzymatic activity, Genetic algorithm, Moisture content, Optimization, Solar energy

\section{Introduction}

From ancient time food conservation played an important role in human history. Drying is probably one of the oldest methods for the sustenance conservation process for numerous agricultural products. It is also one of the most essential operation in many food processing plants which if not done properly will introduce some critical imperfections on the quantity of the final products and the quality as well. Drying procedures require a significant amount of energy in different phases of the operations of producing different output. $60 \%$ of aggregate energy is consumed to generate dry grain in mid-state of the USA (Zomorodian, Zare, Ghasemkhani 2007).

Drying of grain in the tropics is ordinarily accomplished by spreading grains on earth for solar radiation. This technique is moderate and open to situations created by precipitation, winged animals, bugs, dust, and other contaminants. A large portion of farmers can't manage mechanically fueled drying systems. A natural convection solar dryer is an intermediate solution having the advantage of abundant solar energy.

Grain drying is a long delay process which is nonlinear in nature. To achieve the final moisture content is the main purpose of the drying process. The quality of the dried material will be damaged and it will require excessive energy because of over-drying. Again, if the moisture content remains high, the

Address correspondence to Saad Mekhilef, Department of Electrical Engineering, Power Electronics and Renewable Energy Research Laboratory (PEARL), University of Malaya, 50603 Kuala Lumpur, Malaysia.E-mail: saad@um.edu.my

Color versions of one or more of the figures in the article can be found online at www.tandfonline.com/ljge. grains will become vulnerable to mildew. For determining the moisture content there is an option of measuring moisture but due to the inaccuracy of the device this option has some technical limitations. Artificial intelligence methods are better solutions of this problem. By using these methods drying process can be made more accurate.

Granted that numerous approaches to drying have been studied but, no endeavor has been made to optimize the parameters (i.e. the temperature and time) from a financial perspective. Authors in (Klinkman 2013) examined the displaying and optimal configuration of a central solar oriented heating plant with heat storage in the ground. Also, Authors (El-Sebaii et al. 2011; Ghali, Ghaddar, Alsaidi 2011) showed the performance comparison related to the optimized solar air heater. A numerical demonstration for an optimal outline of a solar-assisted drying unit was made by (Janjai et al. 2009; Khatib, Mohamed, Mahmoud, Sopian 2011) for peeled. Applications of artificial intelligence based techniques gained momentum in the past decade. A review was taken by the authors in (Polat and Kirmaci 2012; Prakash and Kumar 2013) where some of them used the Genetic Algorithm (GA). However, their studies did not address the optimal parameter for the drying operation. In addition, authors in (Ttayagarajan et al. 1998) considered the application of GA for optimizing solar system including utilization of the neural networks which feature was to consider the precision and the consistency of the training method. Recently, authors in (Omid, Baharlooei, Ahmadi 2009) proposed simulation of drying kinetics of pistachio nuts. Various topologies to find the optimum control strategy were investigated by them. But they could not draw a final decision about the optimal process. A hybrid control scheme for an air heating system using fuzzy logic and GA was developed by the researchers (Sagar and Kumar 2010). But these researchers used 
a GA only to create the function of the fuzzy logic. In spite of broad research in the field of GA optimization, no study has been aimed to optimize the operating condition. Some studies were done to determine the optimized design of solar dryer utilizing GA, improving the advance dryer condition in drying of grain (Kumar, Jain, and Garg 2010).

This work exhibits the best conditions pertaining to drying materials using an exceptional as well as economical processing of grain. This research suggests a technique according to GA for solving the drying parameter optimization problem. The outcome of this research is a sensible and easy use in food industries.

The structure of this paper is as followed: Evaluation of aspects related to drying engineering as well as applying the GA in drying operation in subsequent portions. Portions following that comprise of GA utilization techniques in this particular platform. Optimization of drying process by the proposed technique in addition to experimental and simulated drying are presented in the second last section and lastly the final outcome with the summary of the potential application is discussed.

\section{Amylase Activity}

$\alpha$-Amylases are starch-degrading enzymes. These are catalysts of the hydrolysis of internal $\alpha-1,4$ d-glycoside bonds in polysaccharides with the retention of $\alpha$-anomeric configuration in products. Most of the $\alpha$-Amylases require calcium ions $\left(\mathrm{Ca}^{2+}\right)$ for their activity, stability and structural integrity. These enzymes have a great effect on the quality of grain. It has an isotropic point 5.3 and an active $\mathrm{pH}$ between 4.8 and 6 as well as an optima temperature of $50^{\circ} \mathrm{C}$ (Shaw et al. 1995). The quality of the grain greatly depends on these enzymes. Researchers in (Morita et al 2004) have revealed that the quality of the grain can be affected by defects in drying process that would affect the acceptability of the commodity to the customers at different stages of the marketing chain. Therefore, a current challenge for rice drying industry is to deliver higher quality products, more efficient post-harvest operation processing method and a prevention of product degradation. The solution of this crisis is the optimization of the drying process.

\section{Method and Material}

\section{Methodology for the Drying Process}

The long grain rough rice has been purchased from a local firm in Malaysia. Grains have rinsed thoroughly for removing the dust and rated by size to eradicate the variations regarding apparent surface area. After that the samples have been sealed in a double layer packet and kept in a refrigerator at $5^{\circ} \mathrm{C}$. This procedure has been done to reach the sample in equilibrium moisture content. Before inserting the sample into the dryer, the samples have been weighted instantaneously. The experiment has been conducted in the open area near the Faculty of engineering in University of Malaya. In the drying process a solar air dryer (SDM-50) has been used. The schematic diagram of the dryer is shown in Figure 1. The dryer is mainly consists of 3 parts, drying chamber, 3 fans providing expected air velocity and electrical heater. A solar panel is attached for operating the fans. With the help of an axial flow blower (100W) the air was flowed.

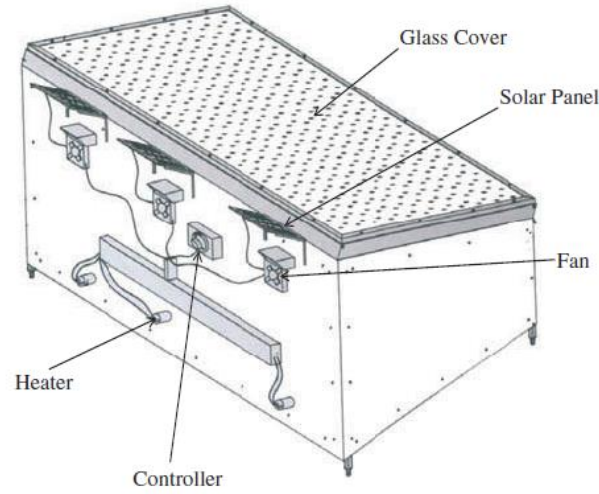

Fig. 1. Schematic diagram of solar powered air dryer.

The air velocity was controlled by the rotating speed of the fan (SPCI-135) and measured by an anemometer (AM-4202). The solar radiation has been measured by the Moll-Goregynstic type pyrometer. The sensitivity of the equipment was $10.5 \mathrm{mV} / \mathrm{Cal}$ $\mathrm{cm}^{2}$ min. Before every test the initial moisture content has been measured by the JSAM Standard (Basunia and Abe 2001). The experiment was conducted under some certain conditions (relative humidity $70 \% \pm 5$ and the average air velocity $1 \mathrm{~m} / \mathrm{s}$ ). And $33^{\circ} \mathrm{C}, 41^{\circ} \mathrm{C}$ and $47^{\circ} \mathrm{C}$ are the three temperature values that were selected as the drying temperatures (see Table 1). These temperatures ensure good amylase activity in grain (Dharmaraj et al. 2013). The grain was placed inside the dryer chamber. Before placing the grain into the dryer chamber the pre-drying weight was measured. The experiments were conducted at intervals of $15 \mathrm{~min}$ each, from $9 \mathrm{hr}$ to $16 \mathrm{hr}$. To measure the weight the samples were collected after each time interval. The moisture content withdrawal from the grain was obtained from the weight difference. The Moisture content of the initial grain found $20 \%$ (w.b). At the temperature $33^{\circ} \mathrm{C}, 41^{\circ} \mathrm{C}$ and $47^{\circ} \mathrm{C}$ drying curves were prepared.

$$
W=\frac{W_{o}}{W_{i}}
$$

Table 1. Experimental Data of Drying Isotherm of Grain

\begin{tabular}{lccc}
\hline \multicolumn{2}{l}{ Drying Temperature } & & \\
\hline $33^{\circ} \mathrm{C}$ & & $41^{\circ} \mathrm{C}$ & $47^{\circ} \mathrm{C}$ \\
$\mathrm{t}(\mathrm{min})$ & $\mathrm{W}_{\exp }$ & $\mathrm{W}_{\exp }$ & $\mathrm{W}_{\exp }$ \\
0 & 1 & 1 & 1 \\
30 & 0.6822 & 0.6663 & 0.6950 \\
60 & 0.6493 & 0.6227 & 0.6408 \\
90 & 0.6325 & 0.6037 & 0.5944 \\
110 & 0.6136 & 0.6000 & 0.5840 \\
120 & 0.5838 & 0.5946 & 0.5454 \\
140 & 0.5691 & 0.5891 & 0.5586 \\
150 & 0.5667 & 0.5715 & 0.5646 \\
160 & 0.5650 & 0.5787 & 0.5638 \\
180 & 0.5638 & 0.5641 & 0.5702 \\
\hline
\end{tabular}


where $W$ weight of sample varied with time. $W_{o}$ Initial weight and $W_{i}$ sample weight at time t.

\section{Quality Experiment}

At first the grains were selected, washed, weighted and carried out to the absorption of moisture from 40 to $45 \%$. After that the grains were carried to germinate in a laboratory scale. The time for the germination was between 4 and 5 days. According to the drying methodology the grains were dried and after that they were stored at $5^{\circ} \mathrm{C}$ (Jayas and White 2003; Dziedzoave et al. 2010; Simsek et al. 2012). The enzymatic activity was determined in every sample according to the literature (Rooney and Wall 2003; Gill, Singh, Singh 2012; Srey, Jahid, Ha 2013). The samples were taken after every $15 \mathrm{~min}$ to measure the enzymatic activity. The content of starch in a sample was represented by SKB per total protein unity (Doehlert and Duke 1983). Using the modified Bradford method the protein concentration of the drying sample was measured (Cai and Chen 2008). From the drying samples $\left(A E_{i}\right)$ amylase specific activities were measured. The specific activity $(A E)$ is a dimensionless value. It was obtained from dividing the specific activity of the samples by specific activity of the initial samples $\left(A E_{o}\right)$.

\section{Modeling of the Drying Process}

This particular model of drying out procedure for the grains mainly includes 2 parts: equation of the moisture diffusion and equation of heat balance. The actual design furthermore incorporates the moisture as well as the heat ranges associated to granules with numerous boundaries similar to diffusion coefficient of moisture, heat along with mass transfer and water activity.

\section{Equation for the Moisture Diffusion}

The generalized system in the diffusion equation of moisture to get a granule is actually written by the subsequent relation.

$$
\frac{\delta \mu_{x} X}{\delta t}=\frac{1}{r^{v}} \frac{\delta}{\delta r}\left(r^{w} \mu_{s} D_{e f} \frac{\delta X}{\delta r}\right)
$$

The feature of the equation of moisture diffusion is partial differential which is nonlinear and the primary constrains are given by

$$
\begin{gathered}
\mathrm{t}=0,0 \leq r \leq R_{d}, X(0, r)=X_{0} \\
t>0,\left[\frac{\delta X}{\delta r}\right]_{r}=0 \\
t>0, r=R_{d}, j_{m, i}=-D \mu_{s}\left[\frac{\delta X}{\delta r}\right]_{r=R_{d}} \\
z_{m, i}=k\left(\mu_{w v, i}-\mu_{w v, g}\right)
\end{gathered}
$$

\section{Equation for the Heat Balance}

The high temperature equalization could be depicted as exchange of heat between inside and outside of the surface of the material. The mathematical statement of heat balance having general geometry is presented by nonlinear partial differential mathematical expression.

$$
\frac{\delta\left[T\left(\rho_{s} C_{p, s}+\mu_{m} c_{p, m}\right)\right]}{\delta t}=\frac{1}{r^{v}} \frac{\delta}{\delta r}\left(r^{v} \sigma \frac{\delta T}{\delta r}\right)
$$

The primary and the boundary conditions are as follows (Chavan, Yakupitiyage, Kumar 2008; Chen, Liang, Guo 2013)

$$
\begin{gathered}
t=0,0 \leq r \leq R_{d} T(0, r)=T_{0} \\
t>0, \frac{\delta T}{\delta r} r_{r=0}=0 \\
t>0, r=R_{d j} j_{T, i}=-\sigma\left[\frac{\delta T}{\delta r}\right]_{r=R_{d}}=\alpha\left[T\left(t, R_{d}\right)-T_{a}\right]+\Delta H_{v}
\end{gathered}
$$

\section{Thin Layer Drying Model}

Different properties like moisture and thermal diffusivity, mass and heat transfer coefficients although in the literature sometimes the drying rate constant is used as their combinations and as a controlling mechanism. The exponential model in Eq. (10) and empirical Page's model in Eq. (11) have been used for describing the drying kinetics of various agricultural products in convective and microwave drying. Both the models were tested for their validity of mushroom under convectional solar drying,

$$
\begin{aligned}
& M R=\exp (-\mathrm{Kt}) \\
& M R=\exp \left(-k t^{n}\right)
\end{aligned}
$$

MR is the moisture ratio, $K$ is drying rate constant $\left(\mathrm{min}^{-1}\right), k$ and $\mathrm{n}$ are the parameter of Page's model, and $t$ is drying time in min. In general moisture ratio (MR) is calculated by this equation,

$$
M R=\frac{M-M_{e}}{M_{0}-M_{e}}
$$

(3) MR is the dimensionless moisture ratio, $M_{\mathrm{t}}$ is the time of moisture content, $M_{0}$ and $M_{\mathrm{e}}$ is the initial and final moisture content at time $t$, on dry basis, respectively.

According to Chin et and Phoungchandang drying data can (4) be used to determine the effective moisture diffusivity, because the $\mathrm{K}$ value depends on the effective moisture diffusivity $\left(D_{e f}\right.$, $\left.\mathrm{m}^{2} / \mathrm{s}\right)$, of the equilibrium moisture $\left(M_{e}\right)$ and half seed thickness $(\mathrm{L}, \mathrm{m})$. This dependence is obtained from diffusion model shown by Eq. (6) adapted from Eq. (5)

$$
K=\frac{p^{2} D_{e f}}{4 L^{2}}
$$




$$
K=\frac{2 D_{e f}}{M_{e} L^{2}}
$$

where $p$ is a value predicted and the half thickness of corn seed used in this work was approximately $3.5 \times 10^{-2} \mathrm{~m}$.

\section{Determination of Multi-Objective Cost Function}

For multi-objective optimization the most important aspect is to determine the objective function. In this work to search the optimal condition of the grain drying process GA was used. Genetic Algorithm for optimization Tool (GAOT) in MATLAB environment was used by many researchers but it is not feasible for the multi-objective optimization as there is a limited option for considering different conditions. Therefore, a program was written for multi-objective optimization. In this program the specific drying conditions (i.e. heat balance, moisture ratio, time and temperature) were specified and the initial population was randomly generated.

The main purpose of the objective function is to minimize the energy consumption and maximize the Amylase activity. To fulfill these objectives together a function was built combining all the desired conditions. The objective function is as follows

$$
\text { Objective Fuction }=-w_{1} \times Q_{T}+w_{2} \times A E
$$

where, $\left(w_{1}+w_{2}\right)=1$ and $w_{1}, w_{2}>0$

$Q_{T}$ represents the consumption of energy and $A E$ is the expression for Amylase activity.

The expression for the required energy for drying is shown below

$$
Q_{T}=\int_{0}^{t} Q_{t} d t
$$

A relationship was established to by the statistical analysis to express the required energy (QT) as a function of Temperature and Moisture ratio. The relation is expressed as follows

$$
\begin{aligned}
Q_{T}= & \int_{0}^{t}\{(3150878-2377 \times T) \\
& +(12725771-9601 \times T) \exp (-23.2 \times M R)\} d t
\end{aligned}
$$

where Temperature $(T)$ and the Moisture ratio $(M R)$ are the control variables.

Temperature $(T)$ represents the dryer temperature and the Moisture ratio $(M R)$ is found from the moisture content of the sample.

Again for maximizing the Amylase activity an expression was established in $\ln A E$ form. The expression is as follows

$$
\begin{aligned}
\ln A E= & -0.6886+0.0476 . \mathrm{t}-1.4522 . \mathrm{T}+0.2910 . t^{2} \\
& +0.7437 T^{2}+0.5209 . \mathrm{t} . \mathrm{T}
\end{aligned}
$$

Where $\mathrm{t}$ is the drying time and $\mathrm{T}$ is the drying temperature.

\section{Application of Genetic Algorithm Technique For Drying Process}

\section{Initialization}

GAs are essentially unique in relation to the optimization algorithm. A GA is a probabilistic system that has established the standards of genetics. In this system the first style is to depict the length of the genetic string named Chromosome. These Chromosomes having a quality of the destination role are termed as Chromosome's fitness. After selecting the length of Chromosome, the initial samples of the Chromosome relies on the arbitrary collection of a number of chromosomes.

In this suggested issue, to determine the values of parameter $T$ (Temperature) and MR (Moisture Ratio) was applied which permitted maximizing moisture ratio, with $\mathrm{MC} \in[22,12]$ and $T \in[30,50]$.

For optimizing the drying process of grain, the GA was designed as follows: The construction of the Chromosome is 30 bits separated into three genes G1, G2 and G3 presenting, MR (Moisture ratio), $T$ (Temperature) and time $(t)$, respectively. From the Eqs. (13) and (14) the particular value of time and temperature is extracted.

$$
M R=M R_{\min }+\left(M R_{\max }-M R_{\min }\right) \times \frac{g 1_{10}}{2^{l}-1}
$$

$$
T=T_{\min }+\left(T_{\max }-T_{\min }\right) \times \frac{g 2_{10}}{2^{l}-1}
$$

and

$$
\mathrm{T}=T_{\min }+\left(T_{\max }-T_{\min }\right) \times \frac{g 2_{10}}{2^{l}-1}
$$

These mathematical statements transforms a binary number into a real number $r \in\left[r_{\min }, r_{\max }\right]$, with an accuracy of $p$ decimal points on the basis of inequality,

$$
2^{k} \geq\left(r_{\max }-r_{\min }\right) \geq 10^{p}
$$

where $p$ is the precision and $k$ is the binary string length.

As an example, two binary string of the Chromosomes are $\mathrm{A} 1$ and $\mathrm{A} 2$ where,

$\mathrm{A} 1=10101$

$\mathrm{A} 2=01110$

Based on the fitness of individual population, a probabilistic choice is made in this stage.

\section{Crossover}

The genetic operator was utilized after the determination operation for generating new results depending on the current results in the sample. Two sorts of essential specialists can be found, one is a crossover and another is mutation. The input of the crossover operation is a couple of Chromosome called Guardians and the output gives a reproduced Chromosome known as Children. Sets associated with individuals were picked up arbitrarily from the mating pool according to the probability of crossover. Provided that the parallel letter set was utilized within GA then every spot 
was given transformation probability from 0 to 1 . The variation in the quality is generally low.

The crossover is done in two steps for selected two Chromosomes. (1) The samples are represented by the binary strings (2) according to the probability distribution, two Chromosomes do crossover in which binary strings can be switched. Therefore single point crossover and two point crossover are used for these Chromosomes. By using uniform probability distribution the crossover point is chosen. As an example, if 3 is the crossover site, the aforementioned A1 and $\mathrm{A} 2$ is changed after the first step crossover and A1 and A2 will be $\mathrm{S} 1=101 \underline{1} \underline{0}$

$\mathrm{S} 2=0 \begin{array}{llll}1 & 1 & 0 & 1\end{array}$

The sample uses the uniform crossover in the first step. In this step 2 binary strings are created and the common terms are searched in both parents, to find out the common mark the common terms. In the string template, the binary bit will be set to 1 when there is matching term in both parents, otherwise it will be zero. Again from the population two numbers are selected randomly and exchanged with each other. As an example, if M1 and $\mathrm{M} 2$ are the set of random number as

$\mathrm{M} 1=131514201711$

M2 = 1711121519101316

And these M1 and M2 is made crossover with B1 and B2, the two parents Chromosomes are changed after the first step crossover. The new children will be like as

$\mathrm{T} 1=01110131514201711$

$\mathrm{T} 2=010011711121519101316$

Second step crossover is done to increase the diversity and the new Chromosomes become

$\mathrm{T} 1=101101711121519101316$

$\mathrm{T} 2=01101131514201711$

Within GA to reach the very least or perhaps greatest position for search engine optimization, progress as well as procedure of selection are usually duplicated iteratively. When the fitness of the best Chromosome does not change significantly, the GA operation is finished.

\section{Mutation}

Changing a small number of bases randomly with small probability is known as mutation. It is performed for maintaining the diversity in the population. To avoid the parameter convergence this operation is essential (Fathi, Mohebbi, Razavi 2011). The proper convergence of GA is dependent upon its configuration which is a very important issue (Aghbashlo et al. 2011).

This convergence is made by defining the control parameters like the population size, the Chromosome size, mutation rate and crossover point. Unfortunately, it is a very difficult task to determine these parameters and in most of the cases, they are defined empirically (Garousi 2008).

From the population, for each selected Chromosome was mutated separately, like the crossover. The operator exchanges with a randomly selected term in the corresponding complimentary subset of the string. In this case a simple point mutation is used. As an example, after mutation

T1 will be as follows:

$\mathrm{T} 1=100101711121519481316$

Parameters for the GA configuration were:
- Size of the population: 200

- Rate of Mutation: 1\%

- Stopping Criteria: 100

\section{Result and Discussion}

\section{Studies for Genetic Algorithm Performance}

A number of experiments were executed to assess the performance of Genetic Algorithm (GA). In each experiment only one variable was varied while keeping the others fixed. Figures from 2 to 4 present the performance of the developed algorithm. After executing the program for 5 times, the average fitness of the performance of GA was obtained in MATLAB (11b).

The performance of the GA was evaluated by the average fitness. Comparison of average fitness after 50, 100 and 200 generations is shown in Figure 2 where it can be clearly seen that Genetic Algorithm converges after 50 generations. Taking more generations does not affect the performance.

The Mutation rate is a very important parameter for GA. Relative performance of genetic algorithm at different mutation rates is particularly shown in Figure 3. It can be easily determined from Figure 2 that the performance of GA decreases while the mutation rate increases. Yet there are many chances of losing many good solutions for high mutation rate. In this work 0.01 or $1 \%$ was set as mutation probability which is considered as a moderate value for minimum disturbance of the best solution.

Performance of GA at different population size is shown in Figure 4. It was expected that with the increment of population size, the performance of the GA will also increase. That is why, 600 individuals ware adopted as population size. This is a moderate size of the population because taking large population need more time to simulate and the give the decisions. This moderate size of a population will ensure the simulation and the decision rapidly in a personal computer

An objective function has been set Eq. (11) considering temperature and moisture content as control variables. The

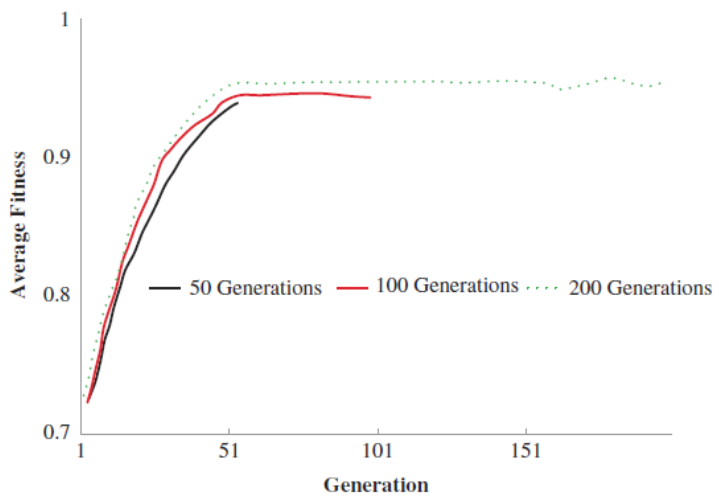

Fig. 2. Performance of Genetic Algorithm (GA) with different numbers of generation. 


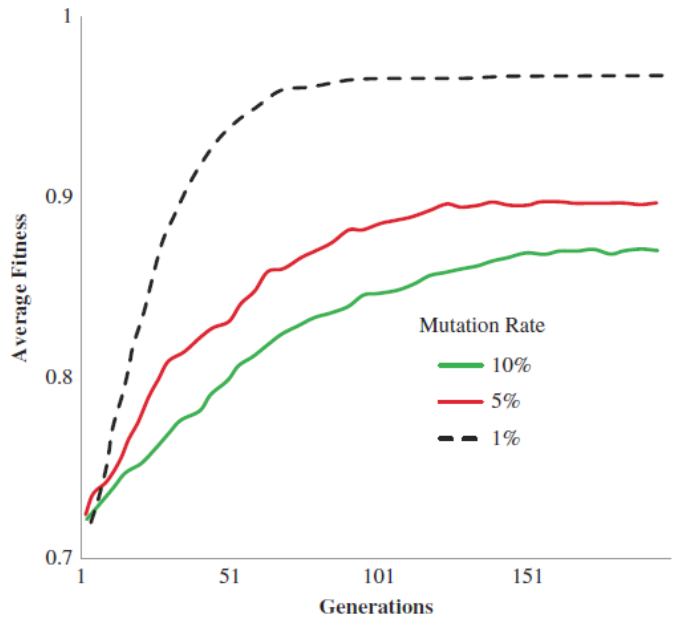

Fig. 3. Performance of Genetic Algorithm (GA) with different mutation rate.

relationship of the objective function with the control variables is shown in Figure 5. The figure represents that the best temperature for drying purpose is $47^{\circ} \mathrm{C}$

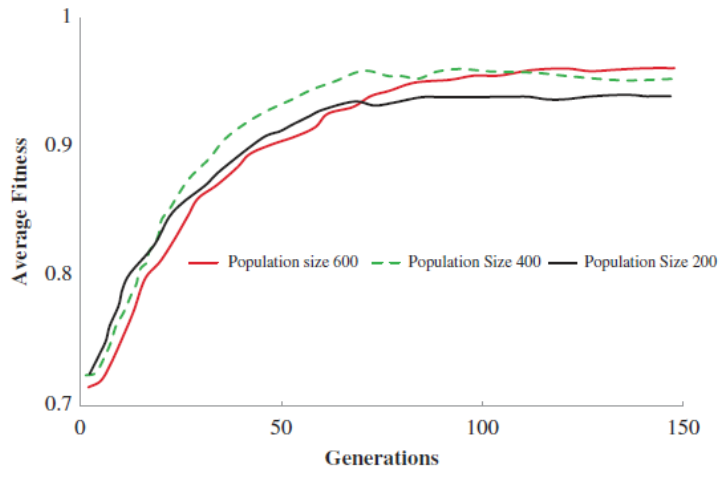

Fig. 4. Performance of Genetic Algorithm (GA) with different population size.

and fungus leading to a minimum loss in nutritive value. The variation of moisture ratio as a function of time is shown in Figure 6.

By the experimental data it is found that to find that the drying curves are parallel to the time axis after $80 \mathrm{~min}$ which indicates the end of drying process because of decreasing of the withdrawal rate of moisture content (Midilli, Kucuk, Yapar 2002).

The constants of Page model are $\mathrm{n}$ and $\mathrm{k}$ where

$$
n=0.68293+0.1094 T+0.017036 R_{H}
$$

\section{Simulated Drying Curve With Experimental Data}

$$
k=0.00561-0.000073 T+0.00169 R_{H}
$$

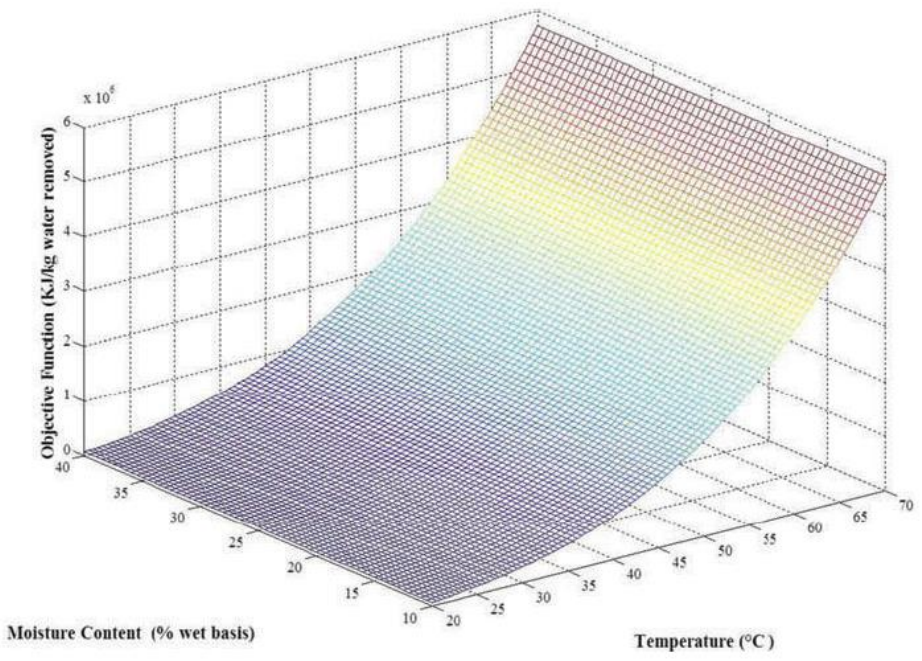

Fig. 5. Relation of objective function with the parameter.

\section{Link to Full-Text Articles :}

\section{http://www.tandfonline.com/doi/pdf/10.1080/15435075.2014.890106}

